\title{
Antimicrobial Resistance of Escherichia coli from Broilers, Pigs, and Cattle in the Greater Kumasi Metropolis, Ghana
}

\author{
Rita Ohene Larbi $\mathbb{D D}^{1,2}$ Linda Aurelia Ofori ${ }^{1}{ }^{1}$, Augustina Angelina Sylverken $\mathbb{D D}^{1,3}$ \\ Matilda Ayim-Akonor $\mathbb{B}^{2}{ }^{2}$ and Kwasi Obiri-Danso ${ }^{1}$ \\ ${ }^{1}$ Department of Theoretical and Applied Biology, Kwame Nkrumah University of Science Technology, Kumasi, Ghana \\ ${ }^{2}$ Animal Health Division, Council for Scientific and Industrial Research-Animal Research Institute, Accra, Ghana \\ ${ }^{3}$ Kumasi Centre for Collaborative Research in Tropical Medicine, PMB, UPO, Kumasi 00233, Ghana
}

Correspondence should be addressed to Rita Ohene Larbi; rolarbi@csir.org.gh

Received 13 April 2021; Accepted 26 May 2021; Published 7 June 2021

Academic Editor: Giuseppe Comi

Copyright (c) 2021 Rita Ohene Larbi et al. This is an open access article distributed under the Creative Commons Attribution License, which permits unrestricted use, distribution, and reproduction in any medium, provided the original work is properly cited.

\begin{abstract}
Globally, resistance to antimicrobial drugs in food animals is on the rise. Escherichia coli of livestock, though commensal in nature, serves as reservoir for antimicrobial resistance genes with the potential of disseminating them. This study sought to examine the antimicrobial resistance profiles of Escherichia coli in broilers, pigs, and cattle in the Kumasi Metropolis and undertake molecular characterisation of the resistances. Faecal E. coli isolates $(n=48)$ were obtained from 10 broiler farms, $(n=43)$ from 15 pig farms, and $(n=42)$ from cattle from the Kumasi Abattoir using standard bacteriological techniques. The Kirby-Bauer disc diffusion method was employed in testing the sensitivities of $133 \mathrm{E}$. coli isolates to 15 antimicrobials. All 48 isolates from broilers presented no resistance to amoxicillin/clavulanic acid and ceftiofur. A 100\% resistance to meropenem was observed in pig and cattle isolates. Multidrug resistance (MDR) across animal groups was 95.8\% $(n=46), 95.3 \%(n=41)$, and $64.3 \%(n=27)$ for broilers, pigs, and cattle, respectively. Twenty-eight isolates presenting phenotypic resistance to aminopenicillins and cephalosporins were screened for the presence of extended-spectrum beta-lactamase (ESBL) genes by PCR. One isolate from poultry and another from cattle tested positive for the bla $\mathrm{CTX}_{\mathrm{C}-\mathrm{M}}$ ESBL gene. There were no positives for the bla $\mathrm{T}_{\mathrm{TEM}}$ and bla $\mathrm{SHV}_{\mathrm{SHV}} \mathrm{ESBL}$ genes. Commensal E. coli of food animal origin represents an important reservoir of antimicrobial resistance that transfers resistance to pathogenic and nonpathogenic microbes affecting humans and animals. There is an urgent need to institute routine surveillance for the establishment of the mechanisms and molecular orientation of resistance in these organisms.
\end{abstract}

\section{Introduction}

The alarming increase in antimicrobial resistance worldwide has triggered responses from major health regulatory bodies. The World Health Organisation (WHO), Food and Agriculture Organisation (FAO), and the World Organisation for Animal Health (OIE) have in recent times laid downstrategic plans to understand and curb the menace which poses a threat to the health and existing drug reserves for treatment of human and animal infections $[1,2]$. A number of factors such as misuse of antimicrobial drugs in human and animal husbandry, sewage and waste water treatment plants, effluents from chemical production factories, and the application of animal manure on farm lands have created conditions for the mingling of human pathogens with environmental bacteria enabling gene mobilization and the spread of antimicrobial resistance [3]. However, antimicrobial resistance may exist naturally in populations that have not previously been exposed to antimicrobials [4].

Escherichia coli, though a commensal in the gut of humans and livestock, serves as a reservoir for antimicrobial resistance genes as they are exposed to the pressures applied on the gut flora of the organism throughout its lifetime [5]. As a result, these strains may acquire certain resistant genes and/or undergo mutations that may enhance the microbial homeostasis in the intestinal environment [6]. These beneficial changes to the commensal microorganism might pose a risk of 
transference of resistant genes to humans through the contamination of meat and other animal products with faecal matter. It is important therefore to monitor the effects of antimicrobial use on the development of resistance in commensals in livestock and selected birds.

Several studies have been conducted in Ghana to assess the extent of antimicrobial resistance [7-9], but only a few have looked at antimicrobial resistance in food animals [10]. This study focused on determining the antimicrobial resistance patterns of commensal $E$. coli in the faeces of broilers, pigs, and cattle to commonly used antibiotics in human and animal husbandry in the Kumasi Metropolis.

\section{Materials and Methods}

2.1. Study Area and Population. This cross-sectional study was carried out from November, 2014, to February, 2016, in Greater Kumasi in the Ashanti region of Ghana. Due to rapid urbanisation of the city, most farms within the metropolis have moved to the periurban communities. To obtain a reliable representative of the target population, the stratified-sampling method was used to divide the Kumasi area into two strata; thus, farms within the metropolis and farms within the periurban towns are to ensure the proper representation of farms within the city and surrounding towns. To this effect, a list of broiler and pig producers were obtained and randomly selected within the two strata. A total of 10 broiler farms and 15 pig farms were involved in the study. The Kumasi Abattoir was employed in the collection of cattle faecal samples as it represented a larger pool of cattle.

2.2. Sample Collection. Three to four fresh droppings from each pen (broilers and pigs) were collected aseptically as a unit into sterile bags. At the abattoir, fresh droppings of cattle kept in a holding pen before slaughter were collected twice a week for three consecutive weeks. All samples were transported under aseptic conditions in a coolbox with ice to the laboratory within 1-2 hours for further analysis.

2.3. Isolation and Identification of E. coli. Five (5) grams of each faecal sample were inoculated into $50 \mathrm{ml}$ of buffered peptone water for preenrichment and resuscitation of bacteria and allowed to sit on the bench at room temperature for about an hour. Direct streaking of the inoculum was made on MacConkey agar (Oxoid, UK) and incubated at $37^{\circ} \mathrm{C}$ for 24 hours. Suspected isolates with pink to red colonies were plated on eosin methylene blue agar (EMBA) (Oxoid, UK) and incubated at $37^{\circ} \mathrm{C}$ for 24 hours. After incubation, isolates with green metallic sheen were subjected to biochemical testing on API 20E (Biomerieux Inc., France).

All confirmed E. coli isolates were put through antimicrobial susceptibility testing by the Kirby-Bauer disc diffusion method with reference to the Clinical Laboratory Standards Institute (CLSI, 2015) standards. Escherichia coli (ATCC 25922) was used as quality control. Nine antimicrobials were tested in broilers: ampicillin $(10 \mu \mathrm{g})$, amoxicillin/clavulanic acid $(30 \mu \mathrm{g})$, streptomycin $(10 \mu \mathrm{g})$, tetracycline $(30 \mu \mathrm{g})$, ciprofloxacin $(5 \mu \mathrm{g})$, nalidixic acid (30 $\mu \mathrm{g})$, ceftiofur (30 $\mu \mathrm{g})$, sulphonamide (300 $\mu \mathrm{g})$, and trimethoprim $(5 \mu \mathrm{g})$.

Eleven antimicrobials were used in pigs and cattle: ampicillin $(10 \mu \mathrm{g})$, tetracycline $(10 \mu \mathrm{g})$, cotrimoxazole $(25 \mu \mathrm{g})$, cefuroxime $(30 \mu \mathrm{g})$, chloramphenicol $(10 \mu \mathrm{g})$, ceftriaxone $(30 \mu \mathrm{g})$, cefotaxime $(30 \mu \mathrm{g})$, ciprofloxacin $(5 \mu \mathrm{g})$, amikacin $(30 \mu \mathrm{g})$, meropenem $(10 \mu \mathrm{g})$, and amoxicillin/ clavulanic $(30 \mu \mathrm{g})$. Antimicrobials tested belonged to these classes: penicillins, quinolones, fluoroquinolones, aminoglycosides, macrolides, cephalosporins, phenicols, folate pathway inhibitors, and tetracyclines.

2.4. Molecular Characterisation of Resistant Isolates. To determine the resistant genes encoding the observed phenotypic resistance, polymerase chain reaction (PCR) was carried out on cephalosporin and aminopenicillin resistant isolates to detect the presence of existing extended-spectrum beta-lactamases (ESBLs). The ESBL primers used were for

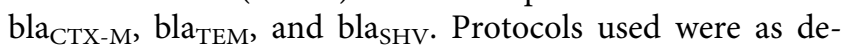
scribed by Hasman et al. [11].

2.5. Data Analysis. Inhibition zones of antimicrobials were interpreted according to CLSI 2015 standards using the WHONET (2015) software. The software was also used to calculate the percentage of multidrug resistance and to determine antimicrobial resistance profiles of isolates. Microsoft Excel (2013) was used to generate other statistical data.

\section{Results}

A total of $133 \mathrm{E}$. coli isolates were obtained from the three animal groups: $36.1 \%(n=48)$ was from broilers, $32.3 \%$ $(n=43)$ from pigs, and $31.2 \%(n=42)$ from cattle.

3.1. Resistance Patterns of Broiler Isolates. All ( $n=48,100 \%)$ isolates from broilers did not show any resistance to amoxicillin/clavulanic acid and ceftiofur (Table 1). The isolates were highly resistant to sulfonamides and tetracycline (95.7\% each), with moderate resistance to nalidixic acid (61.7\%) and ciprofloxacin (23.4\%).

The dominant profile, a combination of ampicillinstreptomycin-tetracycline-sulfonamide-trimethoprim and ciprofloxacin/nalidixic acid, had a total number of 13 isolates representing $25.5 \%$ of broiler isolates tested (Table 2).

3.2. Resistance Patterns of Pig and Cattle Isolates. Of all the antimicrobials tested against faecal $E$. coli from pigs $(n=43)$, complete resistance (100\%) was recorded against meropenem. Ampicillin resistance was recorded among $95.3 \%$ of the isolates. No isolate showed resistance to ceftriaxone and amikacin (Table 3). Two profiles, meropenem-ampicillincefuroxime and meropenem-tetracycline-ampicillin-cefuroxime, had the highest percentage of $18.6 \%$ of pig isolates tested. A total of 17 profiles were observed after testing the faecal isolates against 11 antimicrobials.

Escherichia coli from cattle $(n=42)$ presented no resistance to gentamicin and ciprofloxacin. However, ampicillin 
TABLE 1: Antimicrobial resistance of faecal E. coli from broilers $(n=48)$.

\begin{tabular}{lcccc}
\hline Code & Antimicrobial name & $\% \mathrm{R}$ & $\% \mathrm{I}$ & $\% \mathrm{~S}$ \\
\hline TCY & Tetracycline & 95.7 & 2.1 & 2.1 \\
SSS & Sulfonamides & 95.7 & 0 & 4.3 \\
TMP & Trimethoprim & 93.6 & 0 & 6.4 \\
STR & Streptomycin & 89.4 & 10.6 & 0 \\
AMP & Ampicillin & 80.9 & 4.3 & 14.9 \\
NAL & Nalidixic acid & 61.7 & 14.9 & 23.4 \\
CIP & Ciprofloxacin & 23.4 & 17 & 59.6 \\
AMC & Amoxicillin/clavulanic acid & 0 & 29.8 & 70.2 \\
TIO & Ceftiofur & 0 & 2.1 & 97.9 \\
\hline
\end{tabular}

$\mathrm{R}$, resistant; I, intermediate; $\mathrm{S}$, susceptible.

TABle 2: Resistance profiles of broiler faecal E. coli.

\begin{tabular}{lc}
\hline Resistance profile & \% isolates $(n=48)$ \\
\hline AMP; STR; TCY; SSS; CIP/NAL; TMP & 25.5 \\
AMP; STR; TCY; SSS; TMP; NAL & 17.0 \\
AMP; STR; AMC; TCY; SSS; TMP; NAL & 10.6 \\
AMP; STR; AMC; TCY; SSS; CIP/NAL; TMP & 10.6 \\
AMP; STR; TCY; SSS; TMP & 10.6 \\
AMP; STR; AMC; TCY; SSS; TMP & 4.3 \\
STR; TCY; SSS; CIP/NAL; TMP & 4.3 \\
STR; TCY; SSS; TMP; NAL & 4.3 \\
STR; TCY & 4.3 \\
AMP; STR; TIO; TCY; SSS; TMP; NAL & 2.1 \\
AMP; STR; AMC; SSS; TMP; NAL & 2.1 \\
AMP; STR; AMC; TCY; SSS & 2.1 \\
STR; TCY; SSS; TMP & 2.1 \\
\hline
\end{tabular}

TABLe 3: Antimicrobial resistance of faecal E. coli from pigs $(n=43)$ and cattle $(n=42)$.

\begin{tabular}{|c|c|c|c|c|c|c|c|}
\hline Code & Antimicrobial name & $\% \mathrm{R}$ & $\% \mathrm{I}$ & $\% \mathrm{~S}$ & $\% \mathrm{R}$ & $\% \mathrm{I}$ & $\% \mathrm{~S}$ \\
\hline MEM & Meropenem & 100 & 0 & 0 & 100 & 0 & 0 \\
\hline AMP & Ampicillin & 95.3 & 2.3 & 2.3 & 54.8 & 4.8 & 40.5 \\
\hline TCY & Tetracycline & 44.2 & 16.3 & 39.5 & 26.2 & 33.3 & 40.5 \\
\hline CXM & Cefuroxime & 37.2 & 53.5 & 9.3 & 11.9 & 14.3 & 73.8 \\
\hline SXT & $\begin{array}{l}\text { Trimethoprim/ } \\
\text { sulfamethoxazole }\end{array}$ & 11.6 & 2.3 & 86 & 9.5 & 0 & 9 \\
\hline CHL & Chloramphenicol & 9.3 & 14 & 76.7 & 7.1 & 9.5 & 83.3 \\
\hline GEN & Gentamicin & 7 & 2.3 & 90.7 & 4.8 & 16.7 & 78.6 \\
\hline CTX & Cefotaxime & 7 & 30.2 & 62.8 & 4.8 & 2.4 & 92.9 \\
\hline CIP & Ciprofloxacin & 2.3 & 0 & 97.7 & 2.4 & 0 & 97.6 \\
\hline AMK & Amikacin & 0 & 4.7 & 95.3 & 0 & 7.1 & 92.9 \\
\hline $\mathrm{CRO}$ & Ceftriaxone & 0 & 4.7 & 95.3 & 0 & 0 & 100 \\
\hline
\end{tabular}

$\mathrm{R}$, resistant; I, intermediate; $\mathrm{S}$, susceptible.

resistance was $54.8 \%$, whereas meropenem was $100 \%$ resistant with tetracycline recording $11.9 \%$ resistance (Table 3). 17 profiles were observed in pig faecal isolates (Table 4), whereas cattle isolates had 24 different resistance profiles (Table 5).

3.3. Multidrug Resistance (MDR) in Various Animal Groups. Multidrug resistance (MDR) was defined in our study as resistance to three or more classes of antimicrobials. The three animal groups under study showed distinct patterns of
TABle 4: Resistance profiles of pig faecal E. coli.

\begin{tabular}{lc}
\hline Resistance profile & \% isolates $(n=43)$ \\
\hline MEM; AMP; CXM & 18.6 \\
MEM; TCY; AMP; CXM & 18.6 \\
MEM; SXT; TCY; CTX; AMP; CXM & 9.3 \\
MEM; TCY; CTX; AMP; CXM; CHL & 9.3 \\
MEM; TCY; CTX; AMP; CXM & 9.3 \\
MEM; TCY; AMP; CXM; CHL & 4.7 \\
MEM; GEN; AMP; CXM & 4.7 \\
MEM; AMP & 4.7 \\
MEM; CTX; AMP; CHL & 2.3 \\
MEM; SXT; AMP; CXM & 2.3 \\
MEM; SXT; TCY; AMP & 2.3 \\
MEM; AMK; AMP; CXM & 2.3 \\
MEM; TCY; GEN; AMP; CXM & 2.3 \\
MEM; CIP; AMP; CXM; CHL & 2.3 \\
MEM; GEN; CTX; CRO; CXM; CHL & 2.3 \\
MEM; TCY; CTX; CRO; AMP; CXM & 2.3 \\
MEM; AMK; TCY; CTX; AMP; CXM; CHL & 2.3
\end{tabular}

Table 5: Resistance profiles of cattle faecal E. coli.

\begin{tabular}{lc}
\hline Resistance profile & \% isolates $(n=42)$ \\
\hline MEM; AMP & 14.3 \\
MEM & 9.5 \\
MEM; CXM & 9.5 \\
MEM; AMP; CXM & 9.5 \\
MEM; TCY; CXM & 7.1 \\
MEM; CXM; CHL & 4.8 \\
MEM; TCY; AMP & 4.8 \\
MEM; CTX; AMP; CXM & 4.8 \\
MEM; TCY & 2.4 \\
MEM; AMP; CHL & 2.4 \\
MEM; CRO; AMP & 2.4 \\
MEM; CTX; CXM & 2.4 \\
MEM; TCY; CTX & 2.4 \\
MEM; AMK; CXM & 2.4 \\
MEM; AMP; CXM; CHL & 2.4 \\
MEM; GEN; AMP; CXM & 2.4 \\
MEM; TCY; AMP; CXM & 2.4 \\
MEM; AMK; AMP; CXM; CHL & 2.4 \\
MEM; AMK; CTX; AMP; CXM & 2.4 \\
MEM; SXT; GEN; CTX; AMP; CHL & 2.4 \\
MEM; SXT; TCY; CTX; AMP; CXM & 2.4 \\
MEM; SXT; TCY; CTX; AMP; CXM; CHL & 2.4 \\
MEM; SXT; TCY; GEN; CTX; AMP; CXM & 2.4 \\
\hline
\end{tabular}

MDR with resistance ranging from three to seven antimicrobial classes (Figure 1). Broilers recorded 95.8\% $(n=46)$ MDR, $95.3 \%(n=41)$ for pigs, and $64.3 \%(n=27)$ for cattle (Figure 1).

\section{Detection of ESBL Genes in Aminopenicillin and Cephalosporin Resistant $E$. coli}

Twenty-eight E. coli isolates resistant to ampicillin and cephalosporins were screened for the presence of ESBL genes

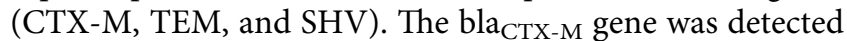
in two $(7.1 \%)$ isolates, one each from cattle $(11.1 \%)$ and 


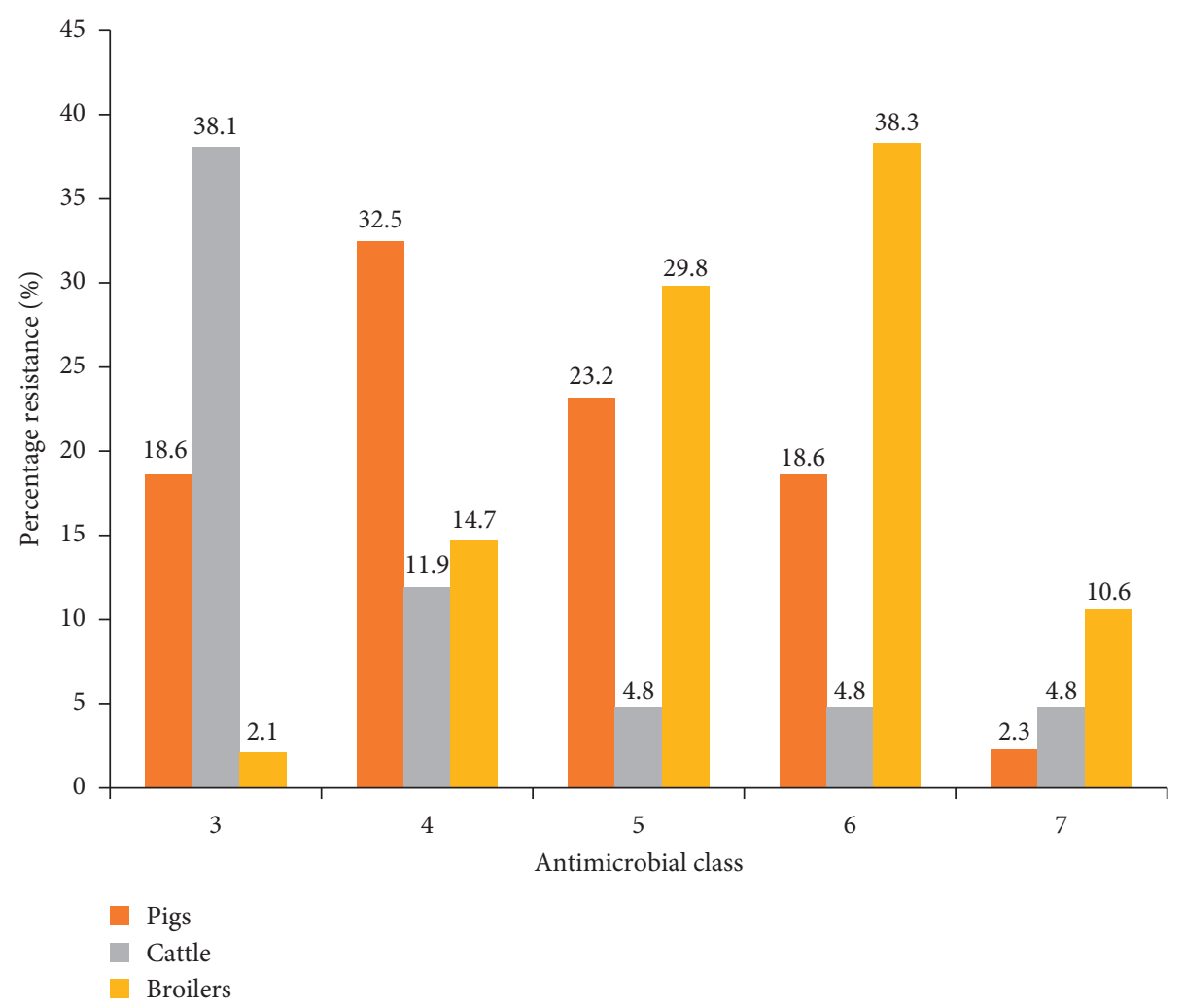

Figure 1: Comparison of percentage multidrug resistance for the three animal groups. Classes tested are penicillins, quinolones, fluoroquinolones, aminoglycosides, macrolides, cephalosporins, phenicols, folate pathway inhibitors, and tetracyclines.

broiler (10\%). There were no positive isolates for the bla $\mathrm{S}_{\mathrm{SHV}}$ and bla $_{\text {TEM }}$ genes tested.

\section{Discussion}

Commensal populations of E. coli are well known to be enriched reservoirs of genetic material made available to nonpathogenic and pathogenic bacteria to pool from [12], posing a threat by way of the development of antimicrobial resistance in animals and humans [13]. In this study, a number of commonly used antimicrobials in humans and animals recorded high resistances.

The tetracyclines recorded a significant level of resistance across all food animal groups in the study. Escherichia coli from broilers showed a resistance of $95.7 \%$, whiles pigs and cattle showed resistance of $44.7 \%$ and $11.9 \%$ respectively. Globally, resistance in the penicillins, tetracyclines, and sulfonamides which are longstanding antimicrobials in animal production is prevalent [14]. The story is not much different from what pertains in Ghana where $[15,16]$ have indicated that tetracycline is the most frequently used antimicrobial in poultry. Therefore, the high level of resistance to tetracycline could be a reflection of the use and misuse of this antimicrobial in poultry.

Escherichia coli resistance observed to ampicillin was high across all three animal groups with $95.7 \%$ of pig isolates being resistant followed by broiler isolates $(80.9 \%)$ and cattle isolates $(54.8 \%)$. The high prevalence may have been as a result of the prolonged use of the drug as well as its long existence in animal husbandry use. There is also the factor of most farmers relying on their experiences as well as inputs from other farmers as compared to patronising the services of veterinary officers $[15,17,18]$ which they consider much more expensive in administering drugs to their animals especially poultry birds; hence, the apparent resistance is found in these two classes of antimicrobials. The moderate resistance in cattle could be linked to the infrequent use of antimicrobials, which are mainly employed for therapeutic purposes in the species [19].

Generally, fluoroquinolone resistance was highest in isolates from broilers $(23.4 \%)$ as compared to those from pigs $(2.4 \%)$ and cattle (0\%). Ciprofloxacin is rarely used in pig farming in Ghana [20]; it is, however, used in the poultry industry. Though not a key antimicrobial drug in animal farming, the high incidence in resistance is a cause for worry because studies have shown a close link between the genomes of certain resistant $E$. coli in humans and those of broilers [21]. Ciprofloxacin and nalidixic acid have in other studies proven to be coresistant; hence, the quinolonenalidixic acid is used as a screening agent for the fluoroquinolone-ciprofloxacin resistance in most studies. A study linked nalidixic acid resistance by disc diffusion in Salmonella typhi strains to high MIC values as much as 10 folds to ciprofloxacin in the same strains [22]. Therefore, it is worthy of note that the resistances presented by ciprofloxacin (disc diffusion) in this study could be comparatively higher when tested with the MIC method, with some sensitive/intermediate isolates possibly showing resistance. 
Quinolone and fluoroquinolone resistance presented could be attributed to the presence of manifold mutations in the quinolone targets of the isolates [23].

The 3rd generation cephalosporin, ceftiofur, designated by FAO as an antimicrobial purposively for veterinary use [24] recorded no resistance in broiler isolates. An accounting factor could be unavailability of this particular drug in the Ghanaian animal farm production. It was, however, an expectation from the start of the study for the possibility of at least observing some level of resistance to cephalosporins. Cefuroxime, a 2nd generation cephalosporin, has been reported to have some level of resistance in Enterobacteriaceae isolated from food animals in Ghana [19]. Cefuroxime as well as cefotaxime (a 3rd generation cephalosporin) resistance was, however, observed in cattle and pigs [25].

The commonest resistance patterns observed were AMP :

STR: TCY : SSS:CIP:TMP:NAL for broilers, MEM: AMP : CXM for pigs, and MEM : AMP for cattle. The antimicrobial classes existing here are the penicillins, streptomycins, fluoroquinolones, quinolones, folate pathway inhibitors, and 2nd generation cephalosporins. Among these are very important human antimicrobial classes (penicillins, fluoroquinolones, and cephalosporins) as such interaction between these microbial populations and human through food/meat consumption and handling of animals (farmers as well as slaughterers) poses a high risk to the human population considering the transfer of resistant genes by mobile genetic elements. Currently, some studies in Ghana have reported microbial contamination of meat products; this could be a source of community-acquired infection if such products are not properly handled and cooked before consumption [26, 27].

Antimicrobial classes were used in comparing multidrug resistance of the food animal groups, and this was defined as isolates having shown resistance to antimicrobials of three or more different antimicrobial classes. Broilers showed the highest percentage of isolates $(95.8 \%)$ with multidrug resistance followed by pigs (95.3\%) and cattle (64.3\%). This finding although lower corresponds to that of Donkor et al. [19] where faecal E. coli of poultry origin recorded a $100 \%$ multidrug resistance. Similar studies carried out in Nigeria [28], Kenya [29], and other parts of the world have also found multidrug resistant bacteria in food animals that can transfer resistance to humans via the foodchain.

Antimicrobial resistance patterns from cattle generally presented the least prevalence $(64.3 \%)$ as compared to the two other animal groups in this study. Antimicrobials are mainly used for therapeutic purposes in cattle and for prophylactic purposes as well, but this is stopped mostly after the cattle have been weaned. Their extensive mode of rearing in Africa, which most likely eliminates the easy transfer of resistance factors between animals, could also account for the low resistances. It has been proven in some studies that calves have higher resistance to antimicrobials than cattle [30], and this explains the results obtained, as mature, ready to slaughter cattle were sampled in this study. In spite of this, some key resistances were observed which included resistance to ampicillin (54.8\%) and cefuroxime $(26.2 \%)$ with meropenem recording a $100 \%$ resistance to all isolates from cattle tested. Carbapenem resistance of Enterobacteriaceae is emerging in humans in Ghana [31, 32], and with the current identification of these trends in animals, much caution must be taken to reduce the transfer of genes at the human animal interface as little is still known about these mechanisms.

ESBL resistance has been recorded in food animals in most parts of Africa, and the most dominant one is the CTX$M$ gene [33, 34]. In Ghana, ESBL genes have been isolated from meat [27]. The possible exchange of these genes between humans and animals may complicate the treatment of common infections in both animals and humans leading to the use of last resort of antimicrobial agents.

\section{Conclusion}

The study has revealed a high incidence of resistance to commonly used antimicrobials in animal husbandry and human medicine. The resulting resistance profiles showed patterns that reflect the use and misuse of antimicrobials in animal farming with the least used or nonexistent antimicrobials presenting low or no resistance to antimicrobials. The study has established that commensal E. coli of food animals is an important reservoir of antimicrobial resistance presenting different facets of resistance due to exposure to antimicrobials as well as acquisition of resistance genes especially through mobile genetic elements. Increased surveillance in food animals must be ensured to create a better picture of the situation and advice policy makers on their decisions.

\section{Data Availability}

All data used in the study are available in the manuscript.

\section{Conflicts of Interest}

The authors declare that they have no conflicts of interest.

\section{Acknowledgments}

The authors are grateful to DANIDA (Danish International Development Assistance) for supporting this research through the Antimicrobial Drug Monitoring and Evaluation of Resistance (ADMER) project. The authors thank Eric Acheampong and Nana Aboagye Acheampong (TAB, KNUST) for technical support. The farmers and abattoir workers who gave their consent for the studies to be conducted are also appreciated.

\section{References}

[1] J. F. Acar, G. Moulin, S. W. Page, and P. Pastoret, "Antimicrobial resistance in animal and public health: introduction and classification of antimicrobial agents," Revue Scientifique et Technique-OIE, vol. 31, no. 1, pp. 15-21, 2012.

[2] WHO, Global Antimicrobial Resistance Surveillance System (GLASS) Report Early Implementation, World Health Organization, Geneva, Switzerland, 2017. 
[3] G. D. Wright, "Antibiotic resistance in the environment: a link to the clinic?" Current Opinion in Microbiology, vol. 13, no. 5, pp. 589-594, 2010.

[4] A. H. Holmes, L. S. P. Moore, A. Sundsfjord et al., "Understanding the mechanisms and drivers of antimicrobial resistance," The Lancet, vol. 387, no. 10014, pp. 176-187, 2016.

[5] B. M. Marshall, D. J. Ochieng, and S. B. Levy, "Commensals: underappreciated reservoir of antibiotic resistance probing the role of commensals in propagating antibiotic resistance should help preserve the efficacy of these critical drugs," Microbe Magazine, vol. 4, no. 5, pp. 231-238, 2009.

[6] G. Cioacata, R. G. Fierro, B. Guerra et al., "The European Union summary report on antimicrobial resistance in zoonotic and indicator bacteria from humans, animals and food in 2016," EFSA Journal, vol. 16, no. 2, p. 5182, 2018.

[7] M. J. Newman, E. Frimpong, E. S. Donkor, J. A. Opintan, and A. Asamoah-Adu, "Resistance to antimicrobial drugs in Ghana," Infection and Drug Resistance, vol. 4, no. 1, pp. 215-220, 2011.

[8] J. Opintan, M. J. Newman, R. E. Arhin, E. S. Donkor, M. Gyansah-Lutterodt, and W. Mills-Pappoe, "Laboratorybased nationwide surveillance of antimicrobial resistance in Ghana," Infection and Drug Resistance, vol. 8, pp. 379-389, 2015.

[9] S. K. Yevutsey, K. O. Buabeng, M. Aikins et al., "Situational analysis of antibiotic use and resistance in Ghana: policy and regulation," BMC Public Health, vol. 17, no. 1, p. 896, 2017.

[10] A. B. Karikari, K. Obiri-Danso, E. H. Frimpong, and K. A. Krogfelt, "Antibiotic resistance of Campylobacter recovered from faeces and carcasses of healthy livestock," BioMed Research International, vol. 2017, Article ID 4091856, 9 pages, 2017.

[11] H. Hasman, D. Mevius, K. Veldman, I. Olesen, and F. M. Aarestrup, “ $\beta$-lactamases among extended-spectrum $\beta$-lactamase (ESBL)-resistant Salmonella from poultry, poultry products and human patients in The Netherlands," Journal of Antimicrobial Chemotherapy, vol. 56, no. 1, pp. 115-121, 2005.

[12] N. B. Shoemaker, H. Vlamakis, K. Hayes, and A. A. Salyers, "Evidence for extensive resistance gene transfer among Bacteroides spp. and among Bacteroides and other genera in the human colon," Applied and Environmental Microbiology, vol. 67 , no. 2, pp. 561-568, 2001.

[13] I. Okeke, S. T. Fayinka, and A. Lamikanra, "Antibiotic resistance in Escherichia coli from Nigerian students, 1986-1998," Emerging Infectious Diseases, vol. 6, no. 4, pp. 393-396, 2000.

[14] T. P. Van Boeckel, J. Pires, R. Silvester et al., "Global trends in antimicrobial resistance in animals in low- and middle-income countries," Science, vol. 365, no. 6459, p. eaaw1944, 2019.

[15] L. A. Andoh, S. Ahmed, J. E. Olsen et al., "Prevalence and characterization of Salmonella among humans in Ghana," Tropical Medicine and Health, vol. 45, no. 1, p. 3, 2017.

[16] V. E. Boamah, C. Agyare, H. Odoi, and A. Dalsgaard, "Practices and factors influencing the use of antibiotics in selected poultry farms in Ghana," Journal of Antimicrobial Agents, vol. 2, no. 2, pp. 2-8, 2016.

[17] F. Adzitey, "Animal and meat production in Ghana-an overview," Journal of World's Poultry Research, vol. 3, no. 1, pp. 1-4, 2013.

[18] M. Ayim-Akonor, R. Krumkamp, J. May, and E. Mertens, "Understanding attitude, practices and knowledge of zoonotic infectious disease risks among poultry farmers in Ghana,"
Veterinary Medicine and Science, vol. 6, no. 3, pp. 631-638, 2020.

[19] E. S. Donkor, M. J. Newman, and D. Yeboah-Manu, "Epidemiological aspects of non-human antibiotic usage and resistance: implications for the control of antibiotic resistance in Ghana," Tropical Medicine \& International Health, vol. 17, no. 4, pp. 462-468, 2012.

[20] J. Osei, "Epidemiology of enterobacteriaceae resistance and prevailing conditions in pig farms in Ashanti region, Ghana," M.Sc. Thesis, University of Pretoria, Pretoria, South Africa, 2013.

[21] T. R. Thorsteinsdottir, G. Haraldsson, V. Fridriksdottir, K. G. Kristinsson, and E. Gunnarsson, "Broiler chickens as source of human fluoroquinolone-resistant Escherichia coli, Iceland," Emerging Infectious Diseases, vol. 16, no. 1, pp. 133-135, 2010.

[22] K. Arti and B. Renuka Das, "Nalidixic acid susceptibility test to screen ciprofloxacin resistance in Salmonella typhi-ProQuest," Indian Journal of Medical Research, vol. 115, pp. 49-54, 2002.

[23] S. S. Namboodiri, J. A. Opintan, R. S. Lijek, M. J. Newman, and I. N. Okeke, "Quinolone resistance in Escherichia coli from Accra, Ghana," BMC Microbiology, vol. 11, no. 1, pp. 1-9, 2011.

[24] R .E. Hornish and S. F. Katarski, "Cephalosporins in veterinary medicine-ceftiofur use in food animals," Current Topics in Medicinal Chemistry, vol. 2, no. 7, pp. 717-731, 2002.

[25] J. Li, Y. Ma, C. Hu et al., "Dissemination of cefotaxime-Mproducing Escherichia coli isolates in poultry farms, but not swine farms, in China," Foodborne Pathogens and Disease, vol. 7, no. 11, pp. 1387-1397, 2010.

[26] F. Adzitey, T. G. Ayum, A. A. Gifty, and A. Samuel, "Microbial quality of chevon and mutton sold in Tamale Metropolis of Northern Ghana," Journal of Applied Sciences and Environmental Management, vol. 14, no. 4, pp. 53-55, 2010.

[27] M. M. Rasmussen, J. A. Opintan, N. Frimodt-Møller, and B. Styrishave, "Beta-lactamase producing Escherichia coli isolates in imported and locally produced chicken meat from Ghana," PLoS One, vol. 10, no. 10, p. e0139706, 2015.

[28] E. O. Adenipekun, C. R. Jackson, A. Oluwadun et al., "Prevalence and antimicrobial resistance in Escherichia coli from food animals in Lagos, Nigeria," Microbial Drug Resistance, vol. 21, no. 3, pp. 358-365, 2015.

[29] L. M. Langata, J. M. Maingi, H. A. Musonye, J. Kiiru, and A. K. Nyamache, "Antimicrobial resistance genes in Salmonella and Escherichia coli isolates from chicken droppings in Nairobi, Kenya," BMC Research Notes, vol. 12, no. 1, p. 22, 2019.

[30] A. R. Khachatryan, D. D. Hancock, T. E. Besser, and D. R. Call, "Role of calf-adapted Escherichia coli in maintenance of antimicrobial drug resistance in dairy calves," Applied and Environmental Microbiology, vol. 70, no. 2, pp. 752-757, 2004.

[31] F. S. Codjoe, C. A. Brown, T. J. Smith, K. Miller, and E. S. Donkor, "Genetic relatedness in carbapenem-resistant isolates from clinical specimens in Ghana using ERIC-PCR technique," PLoS One, vol. 14, no. 9, p. e0222168, 2019.

[32] F. S. Codjoe, Detection and Characterisation of CarbapenemResistant Gram-Negative Bacilli Infections in Ghana, Sheffield Hallam University, Sheffield, UK, 2016.

[33] C. A. Alonso, M. Zarazaga, R. Ben Sallem, A. Jouini, K. Ben Slama, and C. Torres, "Antibiotic resistance in Escherichia coli in husbandry animals: the African perspective," Letters in Applied Microbiology, vol. 64, no. 5, pp. 318-334, 2017. 
[34] D. Fortini, K. Fashae, A. Garcia-Fernandez, L. Villa, and A. Carattoli, "Plasmid-mediated quinolone resistance and -lactamases in Escherichia coli from healthy animals from Nigeria," Journal of Antimicrobial Chemotherapy, vol. 66, no. 6, pp. 1269-1272, 2011. 